\title{
Delayed Leukoencephalopathy: A Rare Complication after Coiling of Cerebral Aneurysms
}

\author{
(D) A. Ikemura, (D) T. Ishibashi, (D) K. Otani, (DI. Yuki, (D). Kodama, (DI. Kan, (D) N. Kato, and (D). Murayama
}

ABSTRACT

BACKGROUND AND PURPOSE: Delayed leukoencephalopathy is a rare complication that occurs after endovascular coiling of cerebral aneurysms. We aimed to describe a clinical picture of delayed leukoencephalopathy and explore potential associations with procedural characteristics.

MATERIALS AND METHODS: We considered endovascular coiling procedures for cerebral aneurysms performed between January 2006 and December 2017 in our institution with follow-up MRIs. We used logistic regression models to estimate the ORs of delayed leukoencephalopathy for each procedural characteristic.

RESULTS: We reviewed 1754 endovascular coiling procedures of 1594 aneurysms. Sixteen of 1722 (0.9\%) procedures demonstrated delayed leukoencephalopathy on follow-up FLAIR MR imaging examinations after a median period of 71.5 days (interquartile range, 30-101 days) in the form of high-signal changes in the white matter at locations remote from the coil mass. Seven patients had headaches or hemiparesis, and 9 patients were asymptomatic. All imaging-associated changes improved subsequently. We found indications suggesting an association between delayed leukoencephalopathy and the number of microcatheters used per procedure $(P=.009)$, along with indications suggesting that these procedures required larger median volumes of contrast medium (225 versus $175 \mathrm{~mL}, \mathrm{OR}=5.5, P=.008$ ) as well as a longer median fluoroscopy duration (123.6 versus 99.3 minutes, OR $=3.0, P=.06$ ). Our data did not suggest that delayed leukoencephalopathy was associated with the number of coils $(P=.57)$, microguidewires $(P=.35)$, and guiding systems $(P=.57)$.

CONCLUSIONS: Delayed leukoencephalopathy after coiling of cerebral aneurysms may have multiple etiologies such as foreign body emboli, contrast-induced encephalopathy, or hypersensitivity reaction to foreign bodies.

ABBREVIATION: DL = delayed leukoencephalopathy

E ndovascular coiling is an effective procedure for preventing rupture of cerebral aneurysms or treating ruptured aneurysms. ${ }^{1,2}$ However, a small percentage of patients experience complications, ${ }^{3}$ which include thromboembolic events (incidence rate of $4.7 \%-12.5 \%$ ) and aneurysm rupture (incidence rate of $2.0 \%-8.8 \%){ }^{3}$ along with posterior reversible encephalopathy

Received September 9, 2019; accepted after revision November 27.

From the Department of Neurosurgery (A.I., T.I., K.O., I.Y., T.K., I.K., N.K., Y.M.), Jikei University School of Medicine, Tokyo, Japan; Siemens Healthcare K.K. (K.O.), Tokyo, Japan; and Department of Neurological Surgery (I.Y.), University of California Irvine, Irvine, California.

Paper presented, in part, at: Annual Meeting of the Japan Neurosurgical Society, October 10-12, 2018, Sendai, Japan; and Annual Meeting of the Japanese Society for Neuroendovascular Therapy, November 22-14, 2018; Migyagi, Japan.

Please address correspondence to Ayako Ikemura, MD, PhD, Department of Neurosurgery, Jikei University School of Medicine, 3-25-8 Nishi-Shimbashi, Minato-ku, Tokyo 105-8461, Japan; e-mail: ayako.udgw613@gmail.com

Indicates article with supplemental on-line tables.

http://dx.doi.org/10.3174/ajnr.A6386 syndrome $e^{4,5}$ and perianeurysmal edema, which may be indicative of symptomatic inflammatory reactions. ${ }^{6-8}$ Recently, delayed leukoencephalopathy (DL) has emerged as a new type of complication under several names, including delayed leukoencephalopathy ${ }^{9}$ delayed enhancing lesions, ${ }^{10}$ and delayed multiple white matter lesions. ${ }^{11}$ Its various suggested etiologies include granulation reaction caused by foreign body emboli from the hydrophilic coating of procedural devices, ${ }^{10,12-15}$ contrast-induced encephalopathy, ${ }^{16,17}$ and nickel ${ }^{11}$ or bioactive polyglycolic/polylactic acid coil sensitivity. ${ }^{9,18}$ Despite an increasing number of reported cases, the overall clinical picture (natural history, incidence, onset time, symptoms, treatment, mortality, and morbidity) of DL remains unclear.

We aimed to define the clinical features of DL and investigate its possible associations with procedural characteristics in a retrospective study of cerebral aneurysms treated by endovascular coiling. 


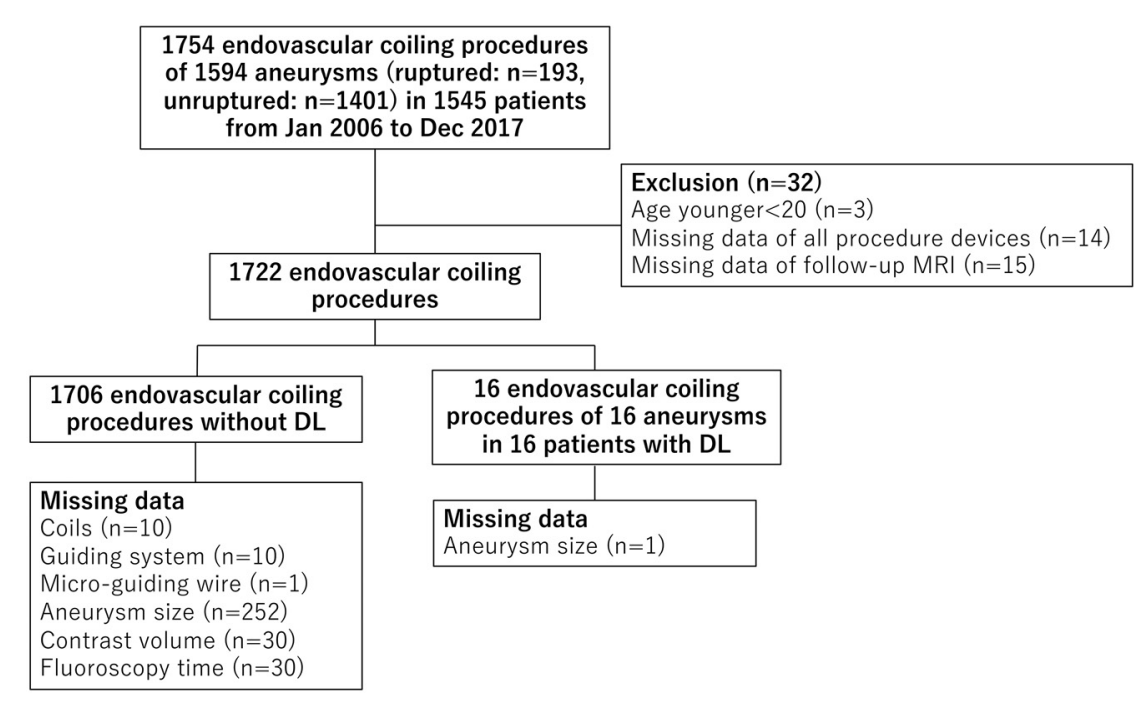

FIG 1. Flow diagram of procedure inclusion. number of devices were customized during each procedure. Double-catheter, balloon-assisted, or stent-assisted techniques were used to prevent coil herniation into the parent artery in cases of wide-neck aneurysms.

\section{MR Imaging}

Follow-up MR imaging examinations were performed using a $1.5 \mathrm{~T}$ or $3 \mathrm{~T}$ MR imaging system, routinely including DWI, FLAIR, T1WI, T2WI, and TOF-MRA to identify possible recanalization of the treated aneurysm or postprocedural complications. Contrastenhanced MR imaging was added at 12 months after the procedure at the discretion of the attending physician.

We considered the following MR

\section{MATERIALS AND METHODS}

\section{Study Design and Participants}

The internal review board of Jikei University Hospital approved this study (approval number 29-228 [8844]). All data related to the management of cerebral aneurysms were extracted from a comprehensive observational data base after approval of an internal review board. Each patient provided comprehensive written consent for the procedures and examinations. Specific informed consent for this study was waived because the data were obtained from routine examinations and treatments and analyzed retrospectively. At the request of the internal review board, we posted a notice of the study and gave patients an opportunity to refuse participation.

Patients with aneurysms (20 years of age or older) who underwent endovascular coiling and a follow-up MR imaging examination after 3, 6, and 12 months at our institution, between January 2006 and December 2017, were considered. For each procedure, patient age and sex, aneurysm location, largest diameter of the aneurysm, fluoroscopy time, and the type and volume of iodinated contrast medium, along with the type and number of devices used in the procedure, were extracted. Devices were defined as guiding systems (ie, guiding sheaths, guiding catheters, and median catheters), microcatheters (including balloon catheters), microguidewires, and coils. We did not consider whether the devices were used simultaneously. Furthermore, we did not consider stents and flow diverters in our analysis. Patients with DL were reviewed for their clinical history, which included MR imaging findings, neurologic findings, treatment of DL, final clinical outcome (minor morbidity, major morbidity, mortality), other procedural complications, allergy tests, time of onset of DL, and time of disappearance of DL.

\section{Procedure}

Endovascular coiling for all aneurysms was performed using digital subtraction angiography with a standard iodine contrast agent following our clinical routine. ${ }^{19,20}$ Patients received general anesthesia and systemic anticoagulation with heparin. The type and imaging outcomes associated with DL:

1. Broad high-signal changes on FLAIR images in the white matter at regions remotely located from the coil mass and observed at $\geq 2$ weeks after the procedure, while the DWI findings remained normal. (These findings were not present immediately after the procedure.)

2. Decreases in size or the disappearance of high-signal changes on FLAIR images within a few months.

\section{Statistical Analyses}

Descriptive statistics were used to summarize the results. Distribution of each variable was assessed using normal quantile plots. DL proportions were calculated for each patient and procedural characteristic. We assessed the association of DL with the number of guiding systems, microguidewires, and microcatheters by classifying the procedures into 2 groups, using ylactic acid coils per procedure was classified as no such coil used and $\geq 1$ such coil used. The association of DL with the variables of age, largest aneurysm diameter, contrast volume, fluoroscopy time, and number of coils was assessed by classifying them into 2 groups of smaller than or equal to or larger than the median of the variable. ORs for the occurrence of DL were estimated by logistic regression. The evidence of an association was assessed using the Fisher exact test. Commercial software was used for the analyses (STATA, Release 15; StataCorp, College Station, Texas).

\section{RESULTS}

\section{Patient Demographics}

Our institution performed 1754 endovascular coiling procedures for 1594 cerebral aneurysms (193 ruptured, 1401 unruptured) between January 2006 and December 2017. The flow diagram regarding the selection of the procedures is presented in Fig 1. Three patients (younger than 20 years of age), 14 procedures that did not have any data regarding procedure devices, and 15 procedures that did not include follow-up MR imaging examination were excluded. There were 1722 procedures in the final analysis. 1 device or $>1$ device. The number of bioactive polyglycolic/pol- 
Table 1: Patients with delayed leukoencephalopathy-aneurysm location, size, detection to disappearance intervals, postprocedural symptoms, treatment, and allergy test

\begin{tabular}{|c|c|c|c|c|c|c|c|}
\hline No. & $\begin{array}{l}\text { Location of } \\
\text { Aneurysm }\end{array}$ & $\begin{array}{l}\text { Largest } \\
\text { Diameter of } \\
\text { Aneurysm } \\
(\mathrm{mm}) \\
\end{array}$ & $\begin{array}{l}\text { Interval between } \\
\text { Intervention and } \\
\text { Detection of DL } \\
\text { (days) }\end{array}$ & $\begin{array}{c}\text { Interval between } \\
\text { Intervention and } \\
\text { Disappearance of } \\
\text { DL (days) }\end{array}$ & Symptom & Treatment & Allergy Test \\
\hline 1 & IC-SHA & NA & 30 & 48 & $\begin{array}{l}\text { Weakness, } \\
\text { aphasia }\end{array}$ & $\begin{array}{c}\text { Free radical } \\
\text { scavenger }\end{array}$ & NA \\
\hline 2 & IC-SHA & 8.4 & 28 & - & Weakness & $\begin{array}{l}\text { Steroid pulse, } \\
\text { antihistamine }\end{array}$ & $\mathrm{Pt}, \mathrm{Zn}, \mathrm{Mn}$ \\
\hline 3 & IC-SHA & 3.8 & 88 & 188 & None & Observation & NA \\
\hline 4 & MCA & 5.7 & 30 & 521 & Weakness & Steroid pulse & All negative \\
\hline 5 & IC-PcomA & 5.1 & 93 & 360 & Headache & Observation & All negative \\
\hline 6 & IC-PcomA & 5.1 & 28 & 224 & None & Observation & Ir \\
\hline 7 & ACA distal & 5.6 & 103 & 269 & None & Observation & NA \\
\hline 8 & IC-ophthalmic & 5.0 & 110 & 200 & None & Observation & $\mathrm{Zn}, \mathrm{Sn}, \mathrm{Cr}$ \\
\hline 9 & IC-ophthalmic & 3.8 & 89 & 230 & None & Observation & $\mathrm{Zn}$ \\
\hline 10 & IC-PcomA & 5.9 & 135 & 366 & None & Observation & NA \\
\hline 11 & IC-cavernous & 17.0 & 55 & - & Weakness & Steroid pulse & All negative \\
\hline 12 & AcomA & 7.9 & 30 & 229 & $\begin{array}{l}\text { Weakness, visual } \\
\text { field loss }\end{array}$ & Steroid pulse & NA \\
\hline 13 & AcomA & 5.0 & 105 & - & None & Observation & NA \\
\hline 14 & IC-PcomA & 9.4 & 48 & 360 & None & Observation & NA \\
\hline 15 & IC-SHA & 5.8 & 16 & 87 & $\begin{array}{l}\text { Weakness, } \\
\text { clumsy hand }\end{array}$ & $\begin{array}{l}\text { Steroid agent, } \\
\text { antihistamine }\end{array}$ & NA \\
\hline 16 & IC-SHA & 14.2 & 99 & 204 & None & Observation & NA \\
\hline
\end{tabular}

Note:-NA indicates not available; IC, internal carotid; SHA, superior hypophysial artery; PcomA, posterior communicating artery; ACA, anterior cerebral artery; AcomA, anterior communicating artery; Pt, platinum; Zn, zinc; Mn, manganese; Ir, iridium; Sn, tin; Cr, chromium; -, imaging changes still slightly remaining at the end of the study.

The DL incidence was $0.9 \%$ (1706 procedures without DL and 16 with DL) during the study duration. Among the 16 aneurysms in the 16 procedures (16 patients) with DL, there were 1 ruptured and 15 unruptured aneurysms. Adjunctive to the endovascular coiling procedure, 1 aneurysm was treated using the stentassisted technique; 1 with the balloon-assisted technique; and 14 with the double-catheter technique. DL patient demographics are presented in Table 1, and the associated details are listed in Online Table 1.

\section{Clinical Course}

Fourteen patients with DL underwent endovascular treatment without intraprocedural complications and were neurologically intact without demonstrating abnormal MR imaging outcomes on the first day postsurgery. Among the 2 other patients with DL, one with an ICA-ophthalmic artery aneurysm demonstrated a worsening of visual acuity and evidence of central retinal artery occlusion. The patient was discharged 5 days after the procedure with mRS 1 (quarter blindness). The other presented with intraoperative aneurysm rupture; however, the coiling procedure could be completed after the rupture. The patient presented with a headache on the next day, and MR imaging revealed a minor subarachnoid hemorrhage. The patient was discharged after 8 days postsurgery without neurologic deficits.

DL symptoms or abnormal MR imaging findings were identified after a median period of 71.5 days (interquartile range, 30101 days). Among the 16 patients with DL, there were 7 symptomatic and 9 asymptomatic patients. Among the symptomatic patients, 7 developed neurologic symptoms, 1 demonstrated continuous headache after discharge, and 6 developed motor weakness ( 1 with aphasia, 1 with visual field loss). These patients recovered from their symptoms after being treated with either steroid therapy or free radical scavenger. The 9 asymptomatic patients recovered without medical intervention. There were no minor or major continuous morbidity or mortality. Among the 16 patients with DL, 7 underwent a skin patch test for metal hypersensitivity and 3 tested negative, while 4 tested positive (Table 1).

\section{MR Imaging Findings}

Follow-up of 4 of the 16 patients with DL included contrastenhanced MR imaging. On the basis of abnormal MR imaging findings, all 16 patients were diagnosed with DL.

A follow-up MR imaging examination for each patient was continued until there was an improvement in the abnormal findings. High-signal changes on FLAIR images for 13 patients disappeared after a median period of 229 days (interquartile range, 200360 days) after undergoing endovascular coiling. Minor levels of high-signal changes persisted in the remaining 3 patients; however, there was a noticeable decrease in the size of the high-signal region.

An illustrative case of a patient with DL whose follow-up included contrast-enhanced MR imaging is presented in Fig 2. DWI outcomes revealed no abnormal changes, but there were extensive high-signal changes on FLAIR images in a region that was primarily perfused by the vessel that underwent the endovascular procedure. Additionally, SWI outcomes demonstrated dotshaped low signals in the regions with high-signal changes.

\section{Procedural Characteristics}

Procedural characteristics are summarized in Table 2 according to DL occurrence; and the associated DL odds ratios for each procedural characteristic, in Table 3. Details regarding the devices used during the procedures of patients with DL are listed in On-line Table 1, as well as the total number of devices by 

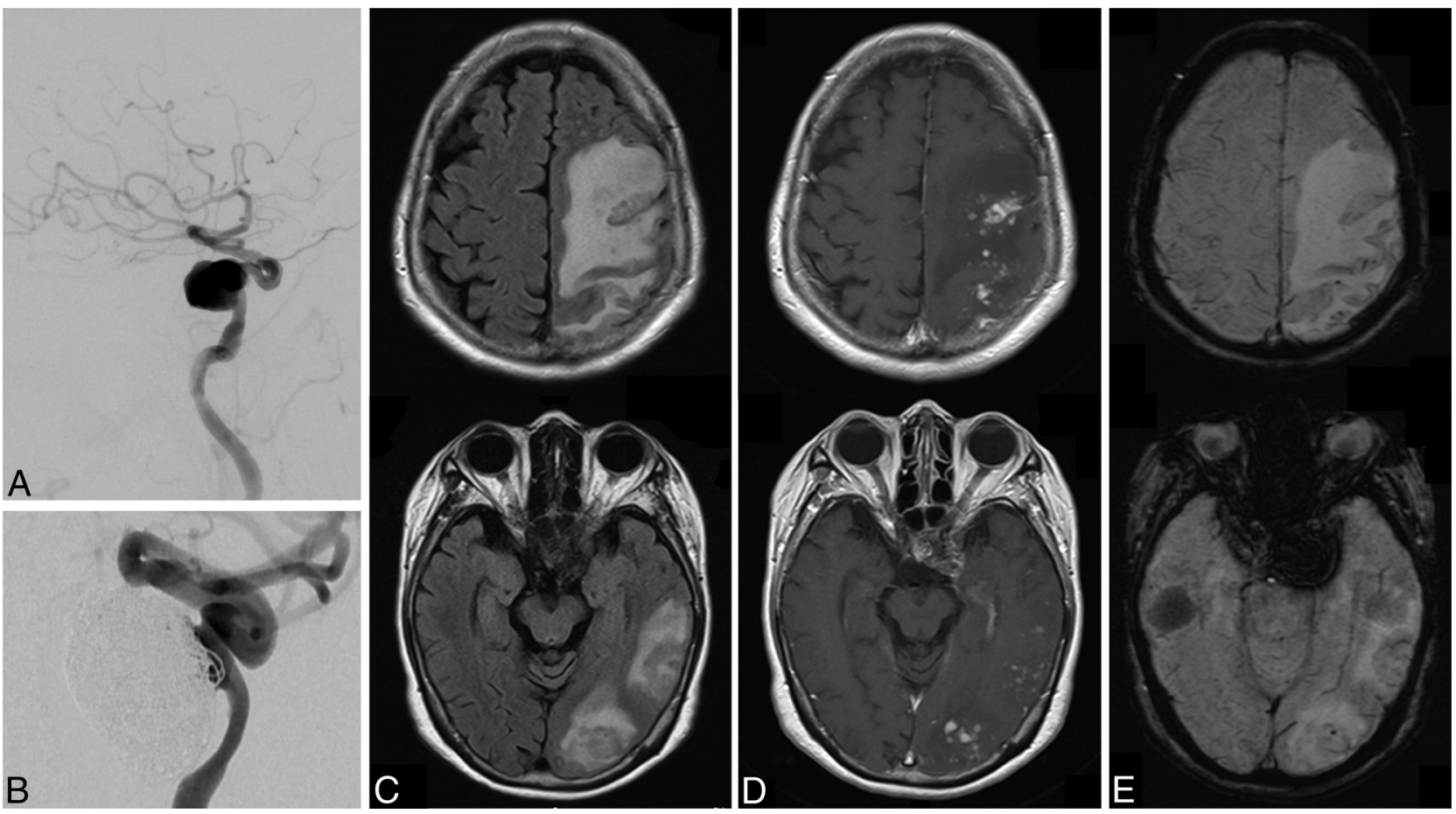

FIG 2. An illustrative case with left ICA aneurysm post-endovascular coiling (patient 11). The aneurysm size is $>17$ mm in maximum diameter ( $A$ ), and endovascular coiling was successfully performed using the double-microcatheter technique (B). After 55 days, right weakness developed and broad high-signal change in the left parieto-occipital region is detected on a FLAIR image $(C)$. In the same region, there is a scattered contrast effect $(D)$ and low spotty regions in the SWI $(E)$. The patient was hospitalized and treated with steroid pulse therapy, and the symptoms and image abnormality subsequently improved.

Table 2: Patient and procedure characteristics (per procedure) with and without $\mathrm{DL}^{\mathrm{a}}$

\begin{tabular}{lccc}
\hline \multicolumn{1}{c}{ Characteristics } & Without DL & With DL & All Patients \\
\hline Female sex & $71.3 \%(1217 / 1706)$ & $81.3 \%(13 / 16)$ & $71.4 \%(1230 / 1722)$ \\
Male sex & $28.7 \%(489 / 1706)$ & $18.8 \%(3 / 16)$ & $28.6 \%(492 / 1722)$ \\
Age $(\mathrm{yr})$ & $59(50-67)$ & $59.5(51-66.5)$ & $59(50-67)$ \\
Largest diameter of aneurysm $(\mathrm{mm})$ & $5.9(4.7-8.0)$ & $5.7(5.0-8.4)$ & $5.9(4.8-8.0)$ \\
Fluoroscopy time $(\mathrm{min})$ & $99.3(72.6-131.0)$ & $123.6(104.8-165.2)$ & $99.5(72.8-131.1)$ \\
Contrast volume $(\mathrm{mL})$ & $175(150-220)$ & $225(195-240)$ & $180(150-220)$ \\
No. of coils & $8(6-13)(\min 1-\max 60)$ & $10.5(6-17)(\min 2-\max 36)$ & $8(6-13)(\min 1-\max 60)$ \\
No. of bioactive polyglycolic/polylactic & $1(0-3)(\min 0-\max 38)$ & $0(0-1)(\min 0-\max 9)$ & $1(0-3)(\min 0-\max 38)$ \\
$\quad$ & & & \\
acid coils & $2(1-2)(\min 1-\max 10)$ & $2(2-2)(\min 2-\max 4)$ & $2(1-2)(\min 1-\max 10)$ \\
$\quad$ & & & $2(2-2)(\min 1-\max 7)$ \\
$\quad$ catheters) & $2(2-2)(\min 1-\max 7)$ & $2(1.5-2.5)(\min 1-\max 3)$ & $2(1-1)$ \\
No. of microguidewires & $1(1-1.5)(\min 1-\max 3)$ & $1(1-1)(\min 1-\max 2)$ & $1(1-1)(\min 1-\max 3)$ \\
No. of guiding systems & &
\end{tabular}

Note:-min indicates minimum; max, maximum.

${ }^{a}$ Categoric variables are expressed in percentage and continuous variables in median and interquartile range.

manufacturer used in our hospital (On-line Table 2, guiding systems; On-line Table 3, microcatheters; On-line Table 4, microguidewires; and On-line Table 5, coils) for the procedures with and without DL. We used a Shuttle sheath (Cook, Bloomington, Indiana) in 2 of the 16 patients with DL, bioactive polyglycolic/ polylactic acid coils in 6 , and 2 microcatheters simultaneously via 1 guiding system in all patients with DL. Use of $>180 \mathrm{~mL}$ of contrast medium was associated with higher $\mathrm{DL}$ odds $(\mathrm{OR}=5.5$; 95\% CI, 1.6-19.5; $P=.008)$ and use of $>1$ microcatheter $(P=.009$, Fisher exact test). Our results also suggested that median fluoroscopy time longer than 99.5 minutes was associated with higher DL odds $(\mathrm{OR}=3.0$; 95\% CI, 1.0-9.5; $P=.06)$. We could not confirm an association of the use of bioactive polyglycolic/polylactic acid coils and higher odds of DL (OR $=0.4 ; 95 \%$ CI, $0.1-1.1 ; P=.08)$.

\section{DISCUSSION}

One of the strengths of our study is the large number of endovascular coiling procedures and patients with DL. We found that $0.9 \%$ of endovascular coiling procedures resulted in patients with DL and that their clinical course was typically benign.

The exact mechanism of DL remains uncertain; however, different hypotheses have been proposed. Some case reports 
Table 3: Odds ratio of complications for each patient and procedure characteristic (per procedure) with and without $\mathrm{DL}^{\mathrm{a}}$

\begin{tabular}{|c|c|c|c|c|}
\hline Characteristics & Without DL & With DL & OR $(95 \% \mathrm{Cl})$ & $P$ Value \\
\hline Female sex & $71.3 \%(1217 / 1706)$ & $81.3 \%(13 / 16)$ & Reference & \\
\hline Male sex & $28.7 \%(489 / 1706)$ & $18.8 \%(3 / 16)$ & $0.6(0.2-2.0)$ & .39 \\
\hline \multicolumn{5}{|l|}{ Age (yr) } \\
\hline 59 or younger & $50.6 \%(863 / 1706)$ & $50.0 \%(8 / 16)$ & Reference & \\
\hline Older than 59 & $49.4 \%(843 / 1706)$ & $50.0 \%(8 / 16)$ & $1.0(0.4-2.7)$ & .96 \\
\hline \multicolumn{5}{|l|}{ Largest diameter of aneurysm } \\
\hline$\leq 5.9 \mathrm{~mm}$ & $49.0 \%(713 / 1455)$ & $53.3 \%(8 / 15)$ & Reference & \\
\hline$>5.9 \mathrm{~mm}$ & $51.0 \%(742 / 1455)$ & $46.7 \%(7 / 15)$ & $0.8(0.3-2.3)$ & .74 \\
\hline \multicolumn{5}{|l|}{ Fluoroscopy time } \\
\hline$\leq 99.5 \mathrm{~min}$ & $50.3 \%(843 / 1676)$ & $25.0 \%(4 / 16)$ & Reference & \\
\hline$>99.5 \mathrm{~min}$ & $49.7 \%(833 / 1676)$ & $75.0 \%(12 / 16)$ & $3.0(1.0-9.5)$ & .06 \\
\hline \multicolumn{5}{|l|}{ Contrast volume } \\
\hline$\leq 180 \mathrm{~mL}$ & $56.2 \%(941 / 1676)$ & $18.8 \%(3 / 16)$ & Reference & \\
\hline$>180 \mathrm{~mL}$ & $43.9 \%(735 / 1676)$ & $81.3 \%(13 / 16)$ & $5.5(1.6-19.5)$ & .008 \\
\hline \multicolumn{5}{|l|}{ No. of coils } \\
\hline$\leq 8$ & $51.0 \%(865 / 1696)$ & $43.8 \%(7 / 16)$ & Reference & \\
\hline$>8$ & $49.0 \%(831 / 1696)$ & $56.3 \%(9 / 16)$ & $1.3(0.5-3.6)$ & .57 \\
\hline \multicolumn{5}{|c|}{$\begin{array}{l}\text { No. of bioactive polyglycolic/polylactic } \\
\text { acid coils }\end{array}$} \\
\hline 0 & $39.9 \%(676 / 1696)$ & $62.5 \%(10 / 16)$ & Reference & \\
\hline$\geq 1$ & $60.1 \%(1020 / 1696)$ & $37.5 \%(6 / 16)$ & $0.4(0.1-1.1)$ & .08 \\
\hline \multicolumn{5}{|c|}{$\begin{array}{l}\text { No. of microcatheters (including balloon } \\
\text { catheters) }\end{array}$} \\
\hline 1 & $27.0 \%(461 / 1706)$ & $0 \%(0 / 16)$ & & .009 (Fisher \\
\hline$>1$ & $73.0 \%(1245 / 1706)$ & $100 \%(16 / 16)$ & & exact test) \\
\hline \multicolumn{5}{|l|}{ No. of microguidewires } \\
\hline 1 & $16.2 \%(276 / 1705)$ & $25.0 \%(4 / 16)$ & Reference & \\
\hline$>1$ & $83.8 \%(1429 / 1705)$ & $75.0 \%(12 / 16)$ & $0.6(0.2-1.8)$ & .35 \\
\hline \multicolumn{5}{|l|}{ No. of guiding systems } \\
\hline 1 & $75.0 \%(1272 / 1696)$ & $81.3 \%(13 / 16)$ & Reference & \\
\hline$>1$ & $25.0 \%(424 / 1696)$ & $18.8 \%(3 / 16)$ & $0.7(0.2-2.4)$ & .57 \\
\hline
\end{tabular}

${ }^{a}$ Categoric variables are expressed in percentage and continuous variables in median and interquartile range.

indicated that DL may be a granulation reaction etiologically associated with foreign body emboli from the hydrophilic coating of the devices. ${ }^{10,12-14}$ Polymer coating embolism from intravascular devices has also been increasingly reported with regard to cardiovascular ${ }^{21}$ and endovascular therapy. ${ }^{22,23}$

In a previous study analyzing a series of 3 cases with delayed enhancing lesions-each treated with the double microcatheter technique-Oh et $\mathrm{al}^{10}$ performed a benchtop evaluation of the inner lumen of microcatheters. They found material wearing off the catheter shafts after multiple in-and-out coil maneuvers and proposed that DL may be caused by the fragmentation of the inner wall of microcatheters and consecutive emboli. Shapiro et $\mathrm{al}^{12}$ reported 5 patients who underwent coil-supported Pipeline Embolization Device (Covidien, Irvine, California) embolization or stent-supported endovascular coiling and presented with foreign body emboli after the procedure. Multiple catheters were used to treat most of the patients evaluated in our study because all endovascular coiling procedures involved stent or flow-diverter deliveries and all patients with DL were treated with multiple microcatheters. Therefore, our results also suggest that the hydrophilic coating of catheters may peel off and act as an embolic source due to the friction between the tight-fitting multiple catheters. The peeling off of the device coating may be associated with the complexity of these procedures, which may be reflected by a tendency of longer fluoroscopy duration in the procedures for patients with DL. In vitro experiments with multiple catheter types and frequent replacement and repositioning are required for further investigation. We routinely used guiding systems with a fairly large inner lumen (up to $8 \mathrm{~F}$ ), but choosing even larger catheters may further lower the friction between devices.

The neurotoxicity of iodinated contrast medium, along with the disruption of the blood-brain barrier and leakage into the brain tissue, has previously been reported to be associated with contrast encephalopathy. ${ }^{16,17,24}$ Contrast-induced encephalopathy typically follows coronary angiography and intracranial endovascular treatment and is characterized on plain CT as leakage of contrast medium. ${ }^{25-27}$ Transient cortical blindness is a commonly reported complication due to contrast-induced encephalopathy and is considered to be associated with high doses of contrast agents. ${ }^{26,28}$ Our study estimated that the odds for experiencing DL were higher for larger volumes of contrast agent $(>180 \mathrm{~mL})$. The patients in our study did not undergo CT examinations; however, 1 patient with DL had high-signal changes on FLAIR images in the hemisphere opposite the treated vessel, and it is possible that the contrast medium may have been translocated to this location. These results support a hypothesis that using a higher dose of contrast medium than the patient can metabolize may be associated with DL. ${ }^{17}$

Nickel allergy or hypersensitivity to polyglycolic/polylactic acid coils is reported to potentially cause DL. ${ }^{9,29}$ In our study, only 7 patients with DL received patch tests for metal hypersensitivity, and none of these 7 patients had metal allergies associated 
with nickel. We used polyglycolic/polylactic acid coils for only 6 of the 16 patients with DL. However, a hypersensitivity reaction to any foreign body used during the invasive procedures continues to be a possible etiology of DL.

It is our understanding that DL may be caused by various factors: foreign body emboli-associated granulation reaction, contrast-induced encephalopathy, or a hypersensitivity reaction to foreign bodies leading to identical image findings. We also cannot exclude interactions and associations between factors such as contrast-induced permeability and the retention of shaved-off catheter coating and the subsequent development of foreign body emboli.

Our study has several limitations. It was a single-center retrospective investigation using data collected from clinical routine procedures. The procedures included stents or flow diverters but not Woven EndoBridge devices (WEB; Sequent Medical, Aliso Viejo, California). There were only a few cases of DL despite the cases being relatively numerous compared with previous studies. None of our patients with DL had posterior circulation aneurysms, which may be related to sample size bias because DL has also been previously reported in such patients. ${ }^{11}$ The number of cases of DL was too few to apply a multivariate logistic regression, and we could not investigate confounding of the procedural characteristics. Pathology tests were not performed. Only a few patients with DL underwent contrast-enhanced MR imaging, and none underwent CT. Our analysis could not distinguish between the simultaneous and consecutive use of microcatheters. We could not investigate associations of specific devices with DL because different types of guiding systems, microcatheters, microguidewires, and coils and their combinations were customized during each procedure, which resulted in unique combinations in almost every procedure.

\section{CONCLUSIONS}

DL is a rare complication following endovascular coiling of cerebral aneurysms. We believe that it may be associated with several etiologies, including foreign body emboli related to complicated procedures or contrast-induced encephalopathy or hypersensitivity reaction to foreign bodies. Although the clinical course of DL is mostly benign, clinicians need to be aware of the potential causes of DL.

\section{ACKNOWLEDGMENTS}

The authors wish to thank Ms Rie Sakima for clinical data preparation and the study team and all the study participants.

Disclosures: Katherine Otani-UNRELATED: Full-time employee of Siemens Healthcare K.K. Katherine Otani supported this study as a biostatistician. Yuichi Murayama-UNRELATED: Consultancy: Stryker, Kaneka Medix; Grants/Grants Pending: Stryker, Siemens K.K., Japan, Comments: research grant*; Payment for Lectures Including Service on Speakers Bureaus: Stryker, Cerenovus, Kaneka Medix; Royalties: Stryker.* *Money paid to the institution.

\section{REFERENCES}

1. Guglielmi G, Vinuela F, Sepetka I, et al. Electrothrombosis of saccular aneurysms via endovascular approach, Part 1: electrochemical basis, technique, and experimental results. J Neurosurg 1991;75:1-7 CrossRef Medline
2. Guglielmi G, Vinuela F, Dion J, et al. Electrothrombosis of saccular aneurysms via endovascular approach, Part 2: preliminary clinical experience. J Neurosurg 1991;75:8-14 CrossRef Medline

3. Orru E, Roccatagliata L, Cester G, et al. Complications of endovascular treatment of cerebral aneurysms. Eur J Radiol 2013;82:1653-58 CrossRef Medline

4. Bartynski WS. Posterior reversible encephalopathy syndrome, Part 1: fundamental imaging and clinical features. AJNR Am J Neuroradiol 2008;29:1036-42 CrossRef Medline

5. Bartynski WS. Posterior reversible encephalopathy syndrome, Part 2: controversies surrounding pathophysiology of vasogenic edema. AJNR Am J Neuroradiol 2008;29:1043-49 CrossRef Medline

6. Craven I, Patel UJ, Gibson A, et al. Symptomatic perianeurysmal edema following bare platinum embolization of a small unruptured cerebral aneurysm. AJNR Am J Neuroradiol 2009;30:19982000 CrossRef Medline

7. Horie N, Kitagawa N, Morikawa M, et al. Progressive perianeurysmal edema induced after endovascular coil embolization: report of three cases and review of the literature. J Neurosurg 2007;106:91620 CrossRef Medline

8. Fanning NF, Willinsky RA, ter Brugge KG. Wall enhancement, edema, and hydrocephalus after endovascular coil occlusion of intradural cerebral aneurysms. J Neurosurg 2008;108:1074-86 CrossRef Medline

9. Fukushima Y, Nakahara I. Delayed leucoencephalopathy after coil embolisation of unruptured cerebral aneurysm. BMJ Case Rep 2018;2018 CrossRef Medline

10. Oh SW, Shin NY, Lee HJ, et al. Delayed enhancing lesions after coil embolization of aneurysms: clinical experience and benchtop analyses. J Neurointerv Surg 2017;9:1243-47 CrossRef Medline

11. Park HS, Nakagawa I, Yokoyama S, et al. Nickel-associated delayed multiple white matter lesions after stent-assisted coil embolization of intracranial unruptured aneurysm. BMJ Case Rep 2017;2017 CrossRef Medline

12. Shapiro M, Ollenschleger MD, Baccin C, et al. Foreign body emboli following cerebrovascular interventions: clinical, radiographic, and histopathologic features. AJNR Am J Neuroradiol 2015;36:2121-26 CrossRef Medline

13. Shotar E, Law-Ye B, Baronnet-Chauvet F, et al. Non-ischemic cerebral enhancing lesions secondary to endovascular aneurysm therapy: nickel allergy or foreign body reaction? Case series and review of the literature. Neuroradiology 2016;58:877-85 CrossRef Medline

14. Fealey ME, Edwards WD, Giannini C, et al. Complications of endovascular polymers associated with vascular introducer sheaths and metallic coils in 3 patients, with literature review. Am J Surg Pathol 2008;32:1310-16 CrossRef Medline

15. Cruz JP, Marotta T, O'Kelly C, et al. Enhancing brain lesions after endovascular treatment of aneurysms. AJNR Am J Neuroradiol 2014;35:1954-58 CrossRef Medline

16. Nagamine Y, Hayashi T, Kakehi Y, et al. Contrast-induced encephalopathy after coil embolization of an unruptured internal carotid artery aneurysm. Intern Med 2014;53:2133-38 CrossRef Medline

17. Leong S, Fanning NF. Persistent neurological deficit from iodinated contrast encephalopathy following intracranial aneurysm coiling: a case report and review of the literature. Interv Neuroradiol 2012;18: 33-41 CrossRef Medline

18. Ulus S, Yakupoğlu A, Kararslan E, et al. Reversible intracranial parenchymal changes in MRI after MCA aneurysm treatment with stent-assisted coiling technique; possible nickel allergy. Neuroradiology 2012;54:897-99 CrossRef Medline

19. Murayama Y, Nien YL, Duckwiler G, et al. Guglielmi detachable coil embolization of cerebral aneurysms: 11 years' experience. $J$ Neurosurg 2003;98:959-66 CrossRef Medline 
20. Murayama Y, Vinuela F, Duckwiler GR, et al. Embolization of incidental cerebral aneurysms by using the Guglielmi detachable coil system. J Neurosurg 1999;90:207-14 CrossRef Medline

21. Chopra AM, Mehta M, Bismuth J, et al. Polymer coating embolism from intravascular medical devices: a clinical literature review. Cardiovasc Pathol 2017;30:45-54 CrossRef Medline

22. Ansari SA, Anderson RR, Caron MJ, et al. Hydrophilic polymer embolic complication during diagnostic cerebral angiography presenting with delayed intracranial hemorrhage: case report and literature review. J Neurointerv Surg 2019;11:80-83 CrossRef Medline

23. Mehta RI, Rai AT, Vos JA, et al. Intrathrombus polymer coating deposition: a pilot study of 91 patients undergoing endovascular therapy for acute large vessel stroke, Part I: histologic frequency. $J$ Neurointerv Surg 2019;11:1191-96 CrossRef Medline

24. Okazaki H, Tanaka K, Shishido T, et al. Disruption of the bloodbrain barrier caused by nonionic contrast medium used for abdominal angiography: CT demonstration. J Comput Assist Tomogr 1989;13:893-95 CrossRef Medline
25. Guimaraens L, Vivas E, Fonnegra A, et al. Transient encephalopathy from angiographic contrast: a rare complication in neurointerventional procedures. Cardiovasc Interv Radiol 2010;33:38388 CrossRef Medline

26. Zwicker JC, Sila CA. MRI findings in a case of transient cortical blindness after cardiac catheterization. Catheter Cardiovasc Interv 2002;57:47-49 CrossRef Medline

27. Dangas G, Monsein LH, Laureno R, et al. Transient contrast encephalopathy after carotid artery stenting. J Endovasc Ther 2001;8: 111-13 CrossRef Medline

28. Niimi Y, Kupersmith MJ, Ahmad S, et al. Cortical blindness, transient and otherwise, associated with detachable coil embolization of intracranial aneurysms. AJNR Am J Neuroradiol 2008;29:603-07 CrossRef Medline

29. Park HS, Nakagawa I, Yokoyama S, et al. Nickel-associated delayed multiple white matter lesions after stent-assisted coil embolization of intracranial unruptured aneurysm. J Neurointerv Surg 2018;10:e1 CrossRef Medline 